\title{
Stability of the High-Pressure Body-Centered-Cubic Phase of Helium
}

\author{
D. Frenkel \\ Department of Physics, Rijksuniversiteit Utrecht, 3508 TA Utrecht, The Netherlands \\ (Received 28 October 1985)
}

\begin{abstract}
This paper reports absolute free-energy calculations of the fluid, body-centered-cubic, and facecentered-cubic phases of helium at $T=327.04 \mathrm{~K}$. We find that at and around this temperature the model potential proposed by Aziz et al. does not yield a stable bec phase. Quantum corrections do not alter this conclusion.
\end{abstract}

PACS numbers: $61.20 . \mathrm{Ja}, 64.60 . \mathrm{Cn}, 67.80 . \mathrm{Gb}$

In 1982 Loubeyre et al. ${ }^{1}$ reported the observation of a cusp in the melting curve of ${ }^{4} \mathrm{He}$ around room temperature. In Ref. 1 and in subsequent publications ${ }^{2-4}$ it is suggested that the cusp in the melting curve is due to the appearance of a thermodynamically stable body-centered cubic (bcc) phase of helium between the fluid and the face-centered cubic (fcc) solid phase, for temperatures above $299 \mathrm{~K}$. Levesque et al. ${ }^{5}$ carried out constant-stress molecular-dynamics simulations ${ }^{6}$ to study the nature of the phase transformations in dense helium. In these simulations the pair potential proposed by Aziz et al. ${ }^{7}$ was used to model the intermolecular interactions in dense helium. Constantstress Monte Carlo simulations on the same model system were carried out by Loubeyre, Levesque, and Weis. ${ }^{8}$ Both sets of simulations indicate that above room temperature the fcc phase of ${ }^{4} \mathrm{He}$ transforms upon expansion (and/or heating) into a bcc solid. The latter phase is observed to melt upon further lowering of the density.

In the present paper I report absolute free-energy calculations of the fcc, bcc, and fluid phases of "Aziz" helium. Our simulation results are fully consistent with the findings of Refs. 6 and 8 on the mechanical stability of the different phases involved. However, I find that the Aziz potential cannot explain the thermodynamical stability of the bcc phase of ${ }^{4} \mathrm{He}$ around 300 $K$. I have also studied the effect of the lowest-order quantum corrections but these have little effect on the relative stability of the fcc and bcc phases.

The excess Helmholtz free energy $F_{\mathrm{ex}}^{\mathrm{n}}(\rho)$ of the fluid phase at density $\rho$ is most easily calculated by thermodynamic integration:

$$
F_{\mathrm{ex}}^{\mathrm{f}}(\rho) / N k T=\int_{0}^{\rho}\left[P\left(\rho^{\prime}\right) / \rho^{\prime} k T-1\right] / \rho^{\prime} d \rho^{\prime} .
$$

In Eq. (1) $P$ is the pressure at density $\rho^{\prime}, T$ is the absolute temperature, and $k$ is the Boltzmann constant. To carry out the integration in Eq. (1) I performed Monte Carlo simulations for a system of 256 particles at a number of densities along the $T=327.04$ isotherm (see Fig. 1). This particular temperature was chosen because the simulations of Loubeyre, Levesque, and Weis ${ }^{8}$ were carried out for the same value of $T$. In Fig.
1 I have included eight state points from Ref. 8. No systematic differences between my data and those of Loubeyre, Levesque, and Weis are observable. In addition, I evaluated the second virial coefficient of Aziz helium at $327.04 \mathrm{~K}$. With use of this information the Monte Carlo data were fitted by a fifth-order polynomial in the density. The free energies of the fcc and bcc solids were evaluated by construction of a reversible path from the solid under consideration to an Einstein crystal with the same structure. ${ }^{9}$ The method used in the present simulations differs slightly from the one described in Ref. 9. Let us consider a poten-

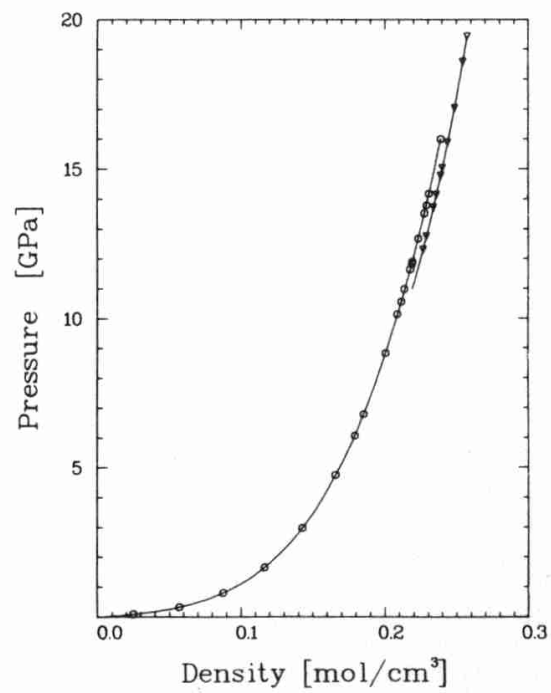

FIG. 1. Equation of state of Aziz helium at $T=327.04 \mathrm{~K}$ Open circles, fluid phase; inverted triangles, fcc phase. Drawn curves, polynomial fits to the Monte Carlo data (see text). The bcc branch has been omitted from this figure. 
tial energy function

$$
\begin{aligned}
U(\lambda)=V_{0}\left(\mathbf{r}_{0}^{N}\right) & +\lambda\left[V\left(\mathbf{r}^{N}\right)-V_{0}\right] \\
& +(1-\lambda) K \sum_{i=1}^{N}\left(\mathbf{r}^{i}-\mathbf{r}_{0}^{i}\right)^{2} .
\end{aligned}
$$

Here $V_{0}$ is the potential energy of the solid with all atoms at their lattice sites, $V(r)$ is the potential energy of an assembly of $N$ particles interacting through the Aziz potential, and $(1-\lambda) K$ is a variable spring constant binding all atoms to their lattice sites. For $\lambda=1$, $U(\lambda)$ describes the interaction of the crystal under consideration; for $\lambda=0, U(\lambda)$ is the potential energy function of an Einstein crystal with the same structure. In practice, $K$ was chosen such that the mean square displacement of the atoms in the Einstein crystal was approximately the same as in the unperturbed solid. As the absolute free energy of the Einstein crystal is known, the free energy of the helium crystal can be determined either by thermodynamic integration, using

$$
\frac{\partial F}{\partial \lambda}=\left\langle V\left(\mathrm{r}^{N}\right)-V_{0}-K \sum_{i=1}^{N}\left(\mathrm{r}^{i}-\mathbf{r}_{0}^{i}\right)^{2}\right\rangle,
$$

or by some other method (see, e.g., Frenkel and Ladd $\left.{ }^{10}\right)$. I computed $F_{\text {ex }}$ for both the fcc and bcc phases of Aziz helium at $\rho_{1} \equiv 0.23966 \mathrm{~mol} / \mathrm{cm}^{3}$. Two methods were employed, namely thermodynamic integration, using a ten-point Gauss-Legendre quadrature, and Bennett's overlapping-distribution method ${ }^{11}$ using twelve values for the coupling constant $\lambda$. Both methods gave identical results to within their respective estimated errors. In what follows I shall be using the thermodynamic integration results for $F_{\mathrm{ex}}^{\mathrm{fcc}}$ and $F_{\mathrm{ex}}^{\mathrm{bcc}}$. All conclusions I shall arrive at below hold also for the overlapping-distribution results. At $\rho_{1}$ I obtain the following excess free energies: $F_{\mathrm{ex}}^{\mathrm{fcc}} / N k T$ $=10.4907 \pm 0.0012$ and $F_{\mathrm{ex}}^{\mathrm{bcc}} / N k T=10.4994 \pm 0.0014$. Note that at $\rho_{1}$ the fcc phase is slightly, but significantly, more stable than the bcc phase. I carried out MC simulations on a 256-particle fcc crystal at seven densities and on a 250-particle bec crystal at five densities. Combining my data with those of Ref. 8 I could fit the fcc and bcc isotherms by a three-term polynomial in the density (see Fig. 2). The finite-size corrections to the excess free energies were estimated to be smaller than the error in the thermodynamic integration results. Combining all the available data we are now in a position to compute the coexistence points of fluid, fcc, and bcc Aziz helium (see Table I). The coexistence lines have been indicated in Fig. 2. Note that we find that at $T=327.04 \mathrm{~K}$, Aziz helium does not have a stable bcc phase. In order for a stable bcc phase to be at all possible at this temperature our estimate of $F_{\mathrm{ex}}^{\mathrm{fcc}}-F_{\mathrm{ex}}^{\mathrm{bcc}}$ has to be off by at least 5 standard deviations (much more, if we use the overlapping- distribution results). I also computed the dependence of the coexistence lines on the temperature using $d P / d T=\Delta H / T \Delta V$. As can be seen from Table I the resulting slopes are very nearly equal. This implies that there is a fairly wide temperature range around $T=327 \mathrm{~K}$ where the bcc phase is not stable.

At first sight the present findings may seem to be at odds with the constant-stress molecular-dynamics and MC simulations of Refs. 5 and 8 . Both sets of simulations report a spontaneous transition from fcc to bcc as the pressure is decreased (or the temperature is increased) and back to $\mathrm{fcc}$ as the pressure is increased (temperature is decreased). There is, however, a considerable amount of hysteresis associated with the transformation $\mathrm{fcc} \rightarrow \mathrm{bcc} \rightarrow \mathrm{fcc}$. In the constant-stress simulations a phase transformation occurs when the initial phase becomes mechanically unstable with respect to the other phase. The phase transformation takes place with an irreversible decrease of the Gibbs free energy of the system. The final state must be

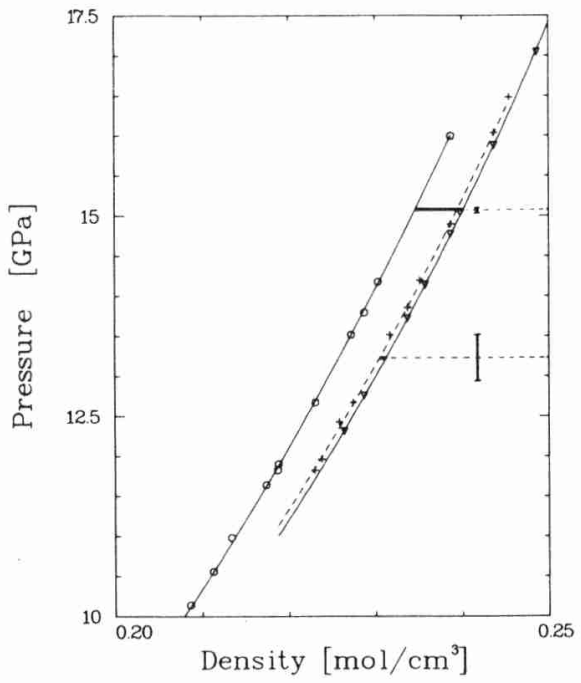

FiG. 2. Equation of state of the fluid (open circles), bcc solid (pluses), and fcc solid (inverted triangles) phases of Aziz helium around its melting point at $T=327.04 \mathrm{~K}$. The drawn and dashed curves are polynomial fits to the Monte Carlo data. The horizontal dashed lines are the classical estimates for the coexistence pressures (see Table 1). Estimated errors in these transition pressure are indicated by vertical bars. Note that this error estimate is almost ten times larger for the fcc-bcc transition than for the fcc-fluid transition. 
TABLE I. Coexistence properties of Aziz helium. The left-hand column shows the classical transition pressure, coexistence densities, and slope of the coexistence line. The right-hand column was obtained from inclusion of quantum corrections to lowest order. Note that the estimated error in the transition pressure is about 10 times larger for the fccbcc transition (lower half) than for the fcc-liquid transition (upper half). The estimated error in the densities is almost exclusively due to the error in the coexistence pressure and has therefore not been indicated separately. Also indicated in the table is the excess Helmholtz free energy of the fcc phase at coexistence. The excess free energy is expressed in units of $N k T$. Knowledge of $F^{\mathrm{ex}}$ of one coexisting phase suffices to compute $F^{\mathrm{ex}}$ of the other. All data in this table were calculated at $T=327.04 \mathrm{~K}$.

\begin{tabular}{|c|c|c|}
\hline & Classical & Quantum $\left[O\left(\hbar^{2}\right)\right]$ \\
\hline$P_{\text {foc-liquid }}(\mathrm{GPa})$ & $15.07(4)$ & $14.73(4)$ \\
\hline$\rho_{\mathrm{fcc}}\left(\mathrm{mol} / \mathrm{cm}^{3}\right)$ & 0.2401 & 0.2355 \\
\hline$\rho_{\text {liq }}\left(\mathrm{mol} / \mathrm{cm}^{3}\right)$ & 0.2345 & 0.2300 \\
\hline$F_{f \infty x}^{\mathcal{F}_{0}} / N k T$ & $10.528(2)$ & $10.501(2)$ \\
\hline$d P / d T(\mathrm{GPa} / \mathrm{K})$ & $0.727(5)$ & $0.737(5)$ \\
\hline$P_{\text {foc }-b c c}(\mathrm{GPa})$ & $13.2(3)$ & $12.9(3)$ \\
\hline$\rho_{\text {fce }}\left(\mathrm{mol} / \mathrm{cm}^{3}\right)$ & 0.2312 & 0.2265 \\
\hline$\rho_{\text {bce }}\left(\mathrm{mol} / \mathrm{cm}^{3}\right)$ & 0.2305 & 0.2258 \\
\hline$F_{\mathrm{fcc}}^{\mathrm{Ex}} / N k T$ & $9.771(2)$ & $9.807(2)$ \\
\hline$d P / d T(\mathrm{GPa} / \mathrm{K})$ & $0.072(7)$ & $0.072(7)$ \\
\hline
\end{tabular}

thermodynamically more stable than the initial state, but it need not be the true equilibrium state at that particular temperature and pressure. In the helium case the constant-stress simulations show a transition from $\mathrm{fcc}$ to bcc. The present calculations indicate that at the point where this transition occurs, the Gibbs free energy of the bcc phase is indeed lower than that of the fcc phase. But both phases are thermodynamically unstable with respect to the liquid. At the thermodynamic melting point of the fcc phase, the bcc phase has a higher Gibbs free energy than the fcc phase. At no point is the bcc phase more stable than both the liquid and the fcc phase. As the bcc phase is compressed in the constant-stress simulations, a transition takes place to the fcc phase. The present results show that this occurs at a point where the fcc phase is indeed thermodynamically stable. Hence the observations made in constant-stress simulations are not in contradiction with the present results. Our absolute free-energy calculations only show that the bcc solid observed in the earlier simulations is not a thermodynamically stable phase.

As ${ }^{4} \mathrm{He}$ is very light, quantum effects might be im- portant even at room temperature. I have therefore computed the lowest-order $\left[O\left(\hbar^{2}\right)\right]$ quantum corrections to the free energy, using the method described by Hansen and Weis. ${ }^{12}$ These quantum corrections were evaluated for the dense liquid and the fcc and bcc solids. From the quantum corrections to the free energy, we obtain the corresponding corrections to the pressure by numerical differentiation. The improved estimates for the coexistence points have been collected in Table I. Although quantum corrections do shift the transitions somewhat, they do not change the qualitative picture. Nor do quantum corrections affect $d P / d T$ much (see Table I).

Hence, we must conclude that the Aziz potential for helium does not predict a stable bcc phase around room temperature. I stress once more that my results are compatible with the simulations of Refs. 6 and 8 because the observed limits of mechanical stability for the fcc and bcc phases are never in conflict with the relative thermodynamical stability that we compute.

I gratefully acknowledge discussions with $\mathrm{M}$. L. Klein, R. leSar, and, in particular, P. Loubeyre, who kindly made the MC data of Ref. 8 available prior to publication.

1P. Loubeyre, J. M. Besson, J. P. Pinceaux, and J. P. Hansen, Phys. Rev. Lett. 49, 1172 (1982).

2J. M. Besson, R. le Toullec, P. Loubeyre, J. P. Pinceaux, and J. P. Hansen, in High Pressure in Science and Technology, edited by C. Homan, R. K. MacCrone, and E. Whalley, Materials Research Society Symposia Proceedings No. 22 (North-Holland, New York, 1984), Vol. 2, p. 13.

${ }^{3}$ P. Loubeyre and J. P. Hansen, Phys. Rev. B 31, 634 (1985).

${ }^{4}$ P. Loubeyre, D. Levesque, and J. J. Weis, to be published.

${ }^{5}$ D. Levesque, J. J. Weis, and M. L. Klein, Phys. Rev. Lett. 51, 670 (1983).

${ }^{6}$ M. Parrinello and A. Rahman, Phys. Rev. Lett. 45, 1196 (1980).

${ }^{7}$ R. Aziz, V. P. S. Nain, J. S. Carley, W. L. Taylor, and G. T. McConville, J. Chem. Phys. 70, 4330 (1979).

${ }^{8} \mathrm{P}$. Loubeyre, D. Levesque, and J. J. Weis, to be published.

${ }^{9}$ D. Frenkel and A. J. C. Ladd, J. Chem. Phys. 81, 3188 (1984).

${ }^{10} \mathrm{D}$. Frenkel, in Proceedings of the International School of Physics "Enrico Fermi," Course 97 (North-Holland, Amsterdam, to be published).

${ }^{11}$ C. H. Bennett, J. Comput. Phys. 22, 245 (1976).

12J. P. Hansen and J. J. Weis, Phys. Rev. 188, 314 (1969) 


\section{Note to Reprint IV.8}

In table I, incorrect values are given for the excess free energy of the face-centred-cubic phase of Aziz helium at coexistence with the body-centred-cubic phase. The correct values are $F_{\mathrm{ex}}^{\mathrm{FCC}} / N k T$ $=9.736(2)$ (classical) and 9.685(2) (quantum mechanical). The latter are the values used in the calculations reported in the paper and the conclusions are therefore unaffected. These errors were brought to light by F.H. Ree. 